\title{
MRI Inter-slice Reconstruction Using Super-Resolution
}

\author{
Hayit Greenspan ${ }^{1}$, Sharon Peled ${ }^{2 \star}$, Gal Oz $^{1}$, and Nahum Kiryati ${ }^{1}$ \\ 1 Faculty of Engineering, Tel Aviv University, Tel Aviv 69978, Israel \\ 2 Department of Radiology, Tel Aviv Sourasky Medical Center, Tel Aviv 64239, Israel
}

\begin{abstract}
MRI reconstruction using super-resolution is presented and shown to improve spatial resolution in cases when spatially-selective RF pulses are used for localization. In 2D multislice MRI, the resolution in the slice direction is often worse than the in-plane resolution. For certain diagnostic imaging applications, isotropic resolution is necessary but true $3 \mathrm{D}$ acquisition methods are not practical. In this case, if the imaging volume is acquired two or more times, with small spatial shifts between acquisitions, combination of the data sets using an iterative superresolution algorithm gives improved resolution and better edge definition in the slice-select direction.
\end{abstract}

\section{Introduction}

Conventional nuclear magnetic resonance imaging utilizes two main methods of encoding the spatial location of nuclear spins contributing to the NMR signal: (1) Spatially selective radio frequency $(\mathrm{RF})$ pulses excite a certain frequency bandwidth. (2) Frequency and phase encoding of the MR signal through temporal variation of the magnetic field gradients before or during signal reception (after the RF excitation). Two-dimensional multislice MR imaging usually relies on selection of slices using the first method, and in-plane encoding using frequency encoding in one direction and phase encoding in the other in-plane direction. The thinner the slice that should be excited by the RF pulse (i.e. the narrower the frequency bandwidth), the longer the duration of the pulse, given the same magnetic field gradient strength. For reasons of RF power deposition, pulse sequence timing, and signal-to-noise ratio (SNR), there are disadvantages to thin-slice excitation.

Three dimensional MR imaging using true 3D data acquisition strategies would be the technique of choice when isotropic spatial resolution is required. Three-dimensional imaging techniques use an initial spatially selective RF pulse to define a "slab" which corresponds to a thick slice, within which thin slices are encoded using phase encoding, while the in-plane encoding is the same as the $2 \mathrm{D}$ method. 3D acquisition methods can be problematic, in terms of the time required for data acquisition, in terms of the achievable contrast, and in terms of image artifacts [1]. For $\mathrm{T}_{1}$-weighted imaging, a 3D fast Fourier-encoding-based

\footnotetext{
* Currently with GE Medical Systems, Israel
} 
gradient-echo sequence is in common use. On the other hand, $\mathrm{T}_{2}$-weighting in a $3 \mathrm{D}$ acquisition can take too long for many clinical situations. Another prominent example in which 3D imaging fails is in echo-planar imaging (EPI) - the underlying technique of choice for functional MRI (fMRI) and diffusion tensor imaging (DTI).

\section{MRI Reconstruction Using Super-Resolution}

The method we present consists of: 1 . The acquisition of a small number of multislice data sets, each volume being shifted in the slice-select direction with respect to the other volumes. 2. The use of super-resolution post-processing in the inter-slice $(\mathrm{z})$ dimension. MRI fourier-encoded in-plane data has a sharp spatial frequency cut-off. This is due to the time limit of the acquisition mechanism and the fact that the information is gathered in the frequency domain (k-space). Thus the best we can do in the $\mathrm{x}$ and $\mathrm{y}$ dimensions is to interpolate the given data to the desired resolution, via zero-padding. The spatial frequencies in the inter-slice direction exhibit a less sharp cut-off thus providing the basis for using a super-resolution algorithm in enhancing the resolution. The iterative backprojection method of Irani-Peleg [2] is used as the super-resolution algorithm in our reconstruction of inter-slice data. This method is based on the minimization of differences between the original low-resolution images, and the low-resolution images that can be generated from down-sampling the current best guess of the high resolution image. The super-resolution algorithm terminates once the error difference between sequential iterations is below a predefined threshold.

\section{Experimental Results}

Experimental results using a "comb"-phantom object are shown in Figure 1. The phantom consists of long thin plastic partitions ("teeth"), lodged in a plastic block, placed $4 \mathrm{~mm}$ apart, surrounded by Gd-DTPA-doped water. The imaging sequence consists of multislice fast spin-echo with 16 slices, $3 \mathrm{~mm}$ thick, approximately parallel to the plastic partitions. Three sets of multislice data were acquired, with $1 \mathrm{~mm}$ shifts in the slice-select direction. Figure 1 shows the results of zero-padding (left) and super-resolution (right). The visibility of the comb teeth has greatly improved by using super-resolution rather than zero-padding interpolation. Similar results for a pomegranate are shown in Figure 2. In both the comb and pomegranate examples, super-resolution brings out features that are inseparable in the source images.

\section{Conclusions}

We propose a novel framework of using super-resolution for MRI resolution augmentation. With inter-slice super-resolution we can effectively break limits on 
slice thickness posed by the physical properties of existing MR imaging hardware. We are currently experimenting with human brain MRI data sets.
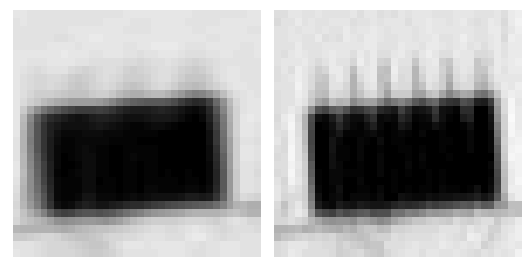

Fig. 1. Super-resolution applied to comb-phantom MRI data. The horizontal axis is the slice-select direction. Left: Low-resolution data with zero-padding interpolation. Right: Super-resolution output.
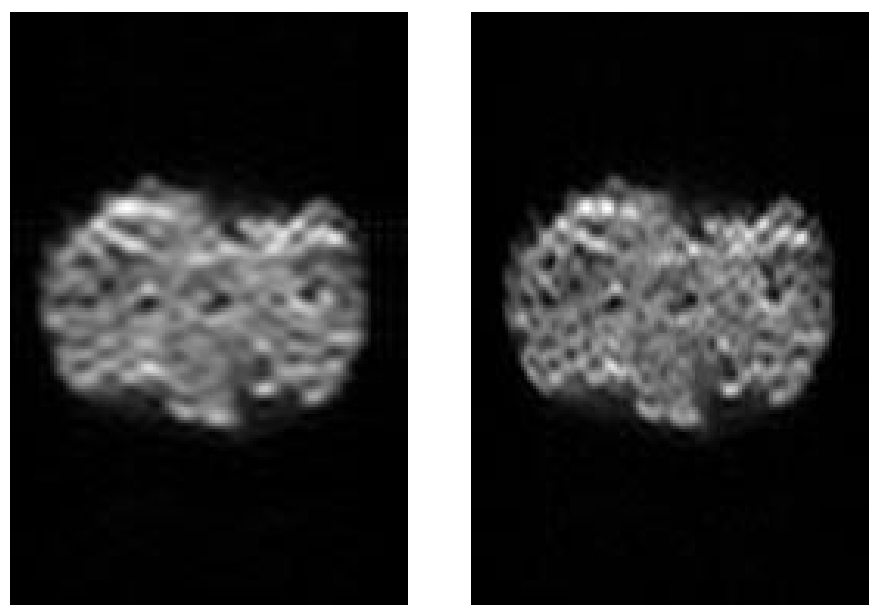

Fig. 2. Super-resolution applied to pomegranate data. The horizontal axis is the slice-select direction. Left: Low-resolution data with zero-padding interpolation. Right: Super-resolution output.

\section{References}

1. Mulkern, R.V., Wong, S.T.S., Winalski, C. and Jolesz, F.A. 1990. "Contrast manipulation and artifact assessment of 2D and 3D RARE sequences". Magn. Reson. Imag. 8:557-566.

2. Irani, M. and Peleg, S. 1993. "Motion analysis for image enhancement: resolution, occlusion and transparency" J. Vis. Comm. Image Rep. 4(4):324-335. 